10 years ESJ

Special edition

\title{
Comportamiento de Compra y Responsabilidad Social de los Estudiantes Universitarios en Tiempos de COVID-19: Ecuador
}

\author{
Mayra Ortega-Vivanco
}

Gabriela Jaramillo-Loaiza

Tangya Tandazo-Arias

Universidad Técnica Particular de Loja, Ecuador

\section{Doi:10.19044/esj.2021.v17n4p80}

Submitted: 28 October 2020

Accepted: 14 December 2020

Published: 07 February 2021
Copyright 2021 Author(s)

Under Creative Commons BY-NC-ND

4.0 OPEN ACCESS

Cite As:

Ortega-Vivanco M., Jaramillo-Loaiza G. \& Tandazo-Arias T. (2021). Comportamiento de Compra y Responsabilidad Social de los Estudiantes Universitarios en Tiempos de COVID19: Ecuador. European Scientific Journal, ESJ, 17(4), 80.

https://doi.org/10.19044/esj.2021.v17n4p80

\section{Resumen}

La investigación analiza la responsabilidad social y el comportamiento de compra de los estudiantes de educación superior del Ecuador, con el propósito de determinar si sus decisiones y elecciones de compras durante la pandemia de la COVID-19 se rigen por factores prioritarios de responsabilidad social. El estudio cuantitativo utiliza el Modelo de Ecuaciones Estructurales (SEM) cuyas variables son: el comportamiento del consumidor, la responsabilidad social, relaciones sociales y factores psicológicos -salud mental y física-. A partir del muestreo por conveniencia, se aplicó 403 encuestas a estudiantes de las universidades públicas y privadas. Los resultados obtenidos para las hipótesis $\mathrm{H}: 1$ son estadísticamente significativos entre el comportamiento del consumidor y factores personales $(0.242, \mathrm{p}<0: 01) ; \mathrm{H} 2$ : comportamiento de compra y responsabilidad social (0.311, p <0:01); y, la H3: comportamiento de compra y salud física y mental $(0.291, \mathrm{p}<0: 01)$. Según la clasificación del tamaño del efecto (f2) propuesta por Cohen, (2013), el modelo presenta una media $\mathrm{f} 2$ en las relaciones probadas de comportamiento del consumidor con las variables relaciones sociales, responsabilidad social y factores psicológicos -salud-, lo que proporciona buena evidencia sobre la 
previsibilidad del modelo.

Palabras clave: Comportamiento De Compra, Responsabilidad Social, Consumo, Estudiantes Universitarios, COVID-19

\title{
Buying Behavior and Social Responsibility of University Students in Times of COVID-19: A Case Study of Ecuador
}

\author{
Mayra Ortega-Vivanco \\ Gabriela Jaramillo-Loaiza \\ Tangya Tandazo-Arias \\ Universidad Técnica Particular de Loja, Ecuador
}

\begin{abstract}
This paper focuses on the social responsibility and purchasing behavior of Ecuadorian higher education students to determine their purchasing decisions and choices during the COVID-19, which is governed by priority factors of sustainability and social responsibility. The quantitative study used the structural equation model (SEM) with the following variables: consumer behavior, social responsibility, social relationships, and psychological factors (mental and physical health). Based on convenience sampling, 403 surveys were carried out with students from public and private universities. The results obtained for hypothesis $\mathrm{H} 1$ is statistically significant between consumer behavior and personal factors (0.242, p <0:01); H2: purchasing behavior and social factors $(0.311, \mathrm{p}<0: 01)$; and $\mathrm{H} 3$ : buying behavior and cultural factors (0.291, p <0:01). According to the classification of the effect size (f2) proposed by Cohen (2013), the model presents a mean $\mathrm{f} 2$ in the proven relationships of consumer behavior with the variables such as social relationships, social responsibility and psychological factors (health), which provides good evidence on the predictability of the model.
\end{abstract}

Keyword: Purchasing behaviour, social responsibility, consumption, university students, COVID-19

\section{Introducción}

Con el pasar de los años se ha evidenciado que la producción y el consumo vigentes son insostenibles. La crisis actual ha develado la importancia de repensar de manera consciente el cambio social que se necesita para re-accionar desde un paradigma que reconozca los límites que tiene la naturaleza, ante la voracidad ilimitada de la satisfacción de necesidades de las personas. 
El distanciamiento físico que el gobierno de Ecuador decretó en el mes de marzo del año 2020, fue una de las medidas que los ciudadanos debían acatar para que, a través del confinamiento en sus hogares, se evitase la propagación del Coronavirus Sars-Covid-2. Más allá del cumplimento o evasión de esta medida, la pandemia reveló la importancia de generar nuevas prácticas de consumo y producción; acelerándose el uso de plataformas digitales.

Ante esta situación, la investigación pretende conocer el comportamiento de compra de aquellos estudiantes que pertenecen a generaciones poblacionales que engloban a consumidores conscientes y responsables. Puntualmente, se identificaron como sujetos de estudio a dos cohortes demográficas, digitales e hiperconectadas, se seleccionaron a jóvenes milénicos y posmilénicos universitarios, quienes generalmente se desenvuelven en medio del crecimiento acelerado de las redes sociales virtuales y se caracterizan por mayor activismo social y medioambiental.

El objetivo principal de este estudio es conocer si durante la pandemia de la COVID-19, este grupo de estudiantes universitarios, al momento de su toma de decisiones de compra, entre los diversos factores sociales y psicológicos, también consideran a la responsabilidad social como uno de los aspectos prioritarios en su comportamiento de consumo sostenible.

Este trabajo de investigación comprende cuatro apartados, el primero presenta algunos referentes teóricos sobre la importancia de nuevos patrones de consumo y producción, responsabilidad social y el comportamiento de compra; el segundo ítem, hace referencia a la metodología, en la que se incluye la muestra, mediciones de las variables e indicadores; y el método de análisis -Modelos de Ecuaciones Estructurales (SEM)-. El tercer apartado, analiza los resultados de las relaciones entre el comportamiento del consumidor medidos a través de factores personales, sociales, y responsabilidad social; y finalmente, se muestran los hallazgos y conclusiones del trabajo.

\section{Marco teórico referencial}

La agenda global 2030, establece entre uno de sus objetivos para el desarrollo sostenible, "Garantizar modalidades de consumo y producción sostenibles". Los avances del crecimiento económico y demográfico que hoy se avizoran, se han generado a través de uno de los costos más altos, la degradación medioambiental, condicionando así, la sostenibilidad del planeta. La pandemia de la COVID-19, mostró la urgencia de mudar de un modelo económico lineal a un modelo circular, en el que se revierta la tendencia de producir-consumir, usar y tirar por nuevos patrones de reducir, reusar y reciclar (Naciones Unidas, 2020).

Específicamente, para determinar cómo las medidas de confinamiento ocasionadas por la COVID-19 impactan en el comportamiento del consumidor 
y responsabilidad social (RS) en los estudiantes universitarios del Ecuador, se consideraron las siguientes hipótesis.

H1: Existe una relación positiva entre los factores sociales y el comportamiento del consumidor;

H2: Existe una relación positiva entre la responsabilidad social y el comportamiento del consumidor; y;

H3: Existe una relación positiva entre los factores psicológicos -salud mental y física- y el comportamiento del consumidor.

Solé (2001) refiere que detrás de la conducta del consumidor, se encuentra el comportamiento de compra; con ello se reafirma la relación entre consumidores y productores dentro del flujo circular de la economía. Para analizar el comportamiento de compra, el presente estudio reconoce que ningún consumidor es igual a otro y que sus diferencias se marcan por la diversidad, caracterizada por factores internos como los recursos del consumidor, motivación, conocimientos, actitudes, personalidad, valores y estilos de vida; y factores externos como la cultura, clase social, influencias personales, familia, situación, estímulos de marketing (Blackwell et al., 2002). De todos ellos, los que aumentan la felicidad, según Caprariello y Reis (2013) son los que poseen propiedad psicológica.

Históricamente, los hábitos de consumo han reflejado cambios según los contextos: social, tecnológico, reglas y regulaciones, desastres naturales y conflictos regionales (Sheth, 2020). Esto permite inferir que el consumo supera la óptica de su análisis netamente económico, porque responde a modos de relación del hombre con sus entornos (Henao \& Córdova, 2007).

En la actualidad, es fundamental que la asimetría de información presente en el mercado se minimice para que los consumidores, más allá de relacionar precio-calidad, incorporen en su decisión de compra verdaderos valores humanos. En ese sentido, para que las empresas oferten sus bienes y servicios bajo principios de honestidad y responsabilidad social, se vuelve trascendental, de la mano de políticas públicas eficientes, la práctica de la responsabilidad individual que dinamicen la responsabilidad organizacional (Rodríguez, 2016).

En relación al comportamiento de compra de las nuevas generaciones Su, C.-H. (Joan), Tsai, C.-H. (Ken), Chen, M.-H., y Lv, W. Q. (2019) evalúan su grado de conciencia ecológica, la percepción de los atributos alimentarios sostenibles y las elecciones alimentarias asociadas con hábitos alimenticios saludables; y, concluyen, que estas generaciones son consumidores con conciencia ecológica. Kuhn y Deetz (2008), Rodríguez y Bustamante (2008), consideran que el consumidor socialmente responsable, es aquel ciudadano que compra bienes y servicios no solo para satisfacer sus necesidades, sino que al momento de tomar decisiones sobre sus compras involucra su 
reposicionamiento, anticipando en sus decisiones -criterio de compra- los efectos sociales y/o medioambientales.

El salto de conciencia social, desde patrones de consumo tradicionales, hacia nuevos modos de consumo responsables, ha llevado a que los consumidores busquen en las opciones que ofrecen las empresas en los mercados, aquellas que dentro de su ADN, incluyan servicios sociales (Kreng \& Huang 2011), que las organizaciones sean sensatas, a través de manifestaciones coherentes entre sus valores y el bienestar de la sociedad (Forte \& Lamont, 1998); deben analizar sus impactos ambientales en sus procesos de producción (Harrison, 2003).

Las nuevas generaciones demográficas son compradores más sensibilizados y mayormente influenciables por los medios (Castelló, Castilla \& Ibáñez, 2005). Por tanto, dentro de las políticas empresariales, además de la rentabilidad, se debe personalizar al consumidor, considerando sus derechos asociados a los del medio ambiente. La práctica de esta responsabilidad corporativa transparente, le permite a la empresa construir confianza y añadir valor. En fin, se trata de una responsabilidad compartida entre todos los actores que confluyen en las sociedades.

\section{Metodología de la investigación \\ Datos y muestra}

La investigación inició los primeros días de junio, durante el período de emergencia sanitaria decretada por el gobierno ecuatoriano (marzo de 2020). El ámbito geográfico es la República del Ecuador y la unidad de análisis los estudiantes universitarios.

El diseño metodológico parte del instrumento del cuestionario (encuesta) compuesta por dos secciones; la primera observa datos generales de los estudiantes universitarios como: ciudad, género, edad, estado civil, tipo de universidad; y modalidad de estudios; mientras que la segunda sección, analiza el comportamiento de compra a través de la construcción de variables relacionadas con los factores de responsabilidad social, factores sociales, y factores psicológicos -salud física y mental.

El cuestionario responde a los elementos relevantes señalados en la literatura sobre el comportamiento del consumidor y los factores personales, responsabilidad social y psicológicos (salud) en tiempo de crisis. En su fase inicial, se construyeron veinte y seis indicadores que conformarán los cinco constructos del Modelo de Ecuaciones Estructurales.

Según Matas y Matas (2018), uno de los instrumentos-escalas más utilizados en las ciencias sociales y estudios de mercado es la escala de Likert, por ello se ha empleado en el estudio, así se definió cinco escalas: 5 (Totalmente de acuerdo), 4 (De acuerdo), 3 (Ni de acuerdo, ni en desacuerdo), 2 (En desacuerdo); y 1 (Totalmente en desacuerdo). 
Por otro lado, Rajennd et al. (2014) y Calder et al. (1981) señalan que las muestras de estudiantes son útiles dentro de las investigaciones para observar la relación entre las diferencias individuales del consumidor y el comportamiento de compra.

El total de encuestas aplicadas vía on-line fueron 403; los cuestionarios se distribuyeron entre estudiantes de las instituciones públicas (55\%) y privadas (45\%), se utilizó el método de muestreo por conveniencia segmentación- con un nivel de confianza del $95 \%$ y un margen de error del $5 \%$.

\section{Mediciones: variables e indicadores}

El Modelo de Ecuaciones Estructurales (SEM) se encuentra conformado por cuatro variables; una variable dependiente, comportamiento de compra; y, tres variables independientes relacionadas con los factores sociales, responsabilidad social y factores psicológicos (salud).

Para examinar los criterios de comportamiento de compra se cuestionó a los participantes sobre el consumo de productos alimenticios básicos, cambios de hábitos, fidelidad de las marcas y uso de internet en sus compras; según (Xiao \& Kim, 2009) los sistemas de valores cambiantes de los consumidores afectan al comportamiento del consumidor y a la satisfacción a través de las variables mediadores de los valores de consumo. Por otro lado, los consumidores asocian sus decisiones de compra con los productos que guardan compatibilidad son sus percepciones simbólicas; es decir que más allá del uso que se pueda hacer de los mismos, se guían por el valor personal que les aportan (Rajennd et al., 2014).

Las relaciones sociales y sostenibilidad en el aislamiento fueron medidas a través de ocho indicadores. Los primeras cinco relacionados con la comunicación online y las redes sociales como plataformas para reconectar con el entorno cercado y ámbito profesional; mientras que las tres variables relacionadas con la sostenibilidad son medidas a través de cuestionamientos relacionados con los medios de movilización, causas sociales, consumo de agua y reducción de plástico, premisas trabajadas por Dagher y Itani (2014), quienes señalan que el comportamiento del consumo individual afecta al medio ambiente y plantean la necesidad que los consumidores participen en comportamientos amigables y estrategias ecológicas.

Finalmente, el factor psicológico fue medido a través de cuatro indicadores relacionados con el ejercicio y salud mental, humor, y compra de seguros. Cao et al. (2020) investigaron cómo una emoción positiva discreta impacta las decisiones de los consumidores sobre las discriminaciones de los productos saludables versus los no saludables. Por otro lado, Li y Atkinson, (2020) examinan el efecto de la propiedad psicológica sobre la felicidad del 
consumidor, medida a través de la satisfacción de las necesidades psicológicas básicas.

\section{Método de análisis}

Para cumplir con el objetivo de la investigación y comprobar las hipótesis planteadas, se utilizó el Modelo de Ecuaciones Estructurales (SEM), caracterizado por ofrecer alta confiabilidad y validez a las investigaciones inmersas en el campo empresarial y ciencias sociales (Henseler et al., 2016).

Los modelos SEM, son una familia de modelos estadísticos multivariantes que permiten estimar el efecto y las relaciones entre múltiples variables. Estos modelos surgieron por la necesidad de dar mayor flexibilidad a los modelos de regresión, pues son menos restrictivos y permiten incluir errores de medida tanto en las variables dependientes como en las variables independientes, de ahí su complejidad y uso extendido.

El SEM es utilizado para analizar variables con relación de dependencia, y múltiples relaciones entre variables independientes, combina dos técnicas estadísticas: análisis factorial y regresión. Conformado por constructos o caminos-elementos no observables representados por múltiples indicadores y por las relaciones de dependencia entre constructos (Hair, Black, Babin \& Anderson, s. f.). Este modelo permite la combinación y la contrastación entre el conocimiento a priori y las hipótesis a través de datos empíricos, por lo que, generalmente se lo tipifica como confirmatorio, antes que exploratorio (Haenlein \& Kaplan, 2004).

La modelización de ecuaciones estructurales es una metodología estadística que permite utilizar un observatorio múltiple para la cuantificación y la prueba de teorías científicas; se precisan diferentes interacciones entre las variables latentes (exógenas y endógenas), los parámetros a estimar, las matrices y los errores a considerar. En fin, permiten modelar y probar fenómenos complejos, con rigor estadístico (Schumacker \& Lomax, 2010). Determinan la relación de dependencia o de independencia que tienen las variables que intervienen en estos modelos a través de la integración de ecuaciones lineales (Afifi \& Clark, 1990).

Así, el modelo fue construido por tres variables independientes y diez indicadores que medirán los factores personales (relaciones sociales), consumo y responsabilidad social y aspectos psicológicos (salud) y; cuatro indicadores que observan el comportamiento de compra durante el confinamiento (ver Tabla 1).

Tabla 1. Constructos e indicadores del modelo

\begin{tabular}{|c|c|c|c|}
\hline Variables & Código & Indicadores & $\begin{array}{c}\text { Nro. } \\
\text { Indicadores }\end{array}$ \\
\hline $\begin{array}{c}\text { Comportamiento } \\
\text { de compra }\end{array}$ & CCali_01 & $\begin{array}{c}\text { Cambios en las costumbres de } \\
\text { alimentación }\end{array}$ & 4 \\
\hline
\end{tabular}




\begin{tabular}{|c|c|c|c|}
\hline & CCplo_02 & $\begin{array}{l}\text { Preferencias en el consumo de } \\
\text { productos locales }\end{array}$ & \\
\hline & CCsan_03 & $\begin{array}{l}\text { Alimentación sana antes del } \\
\text { confinamiento }\end{array}$ & \\
\hline & CCfa_04 & $\begin{array}{c}\text { Consumo de productos de } \\
\text { primera necesidad relacionado } \\
\text { con la preferencia familiar }\end{array}$ & \\
\hline \multirow[t]{4}{*}{$\begin{array}{l}\text { Relaciones } \\
\text { sociales }\end{array}$} & CScom_01 & $\begin{array}{l}\text { Comunicación online y redes } \\
\text { sociales como plataformas para } \\
\text { reconectar con el entorno } \\
\text { cercano }\end{array}$ & \multirow[t]{4}{*}{4} \\
\hline & CSwhat_02 & $\begin{array}{c}\text { Uso de aplicaciones de } \\
\text { mensajería como WhatsApp }\end{array}$ & \\
\hline & CSrea_03 & $\begin{array}{c}\text { Uso de aplicaciones de } \\
\text { videoconferencia (Zoom, } \\
\text { Google, Classroom, Microsoft } \\
\text { Teams) en actividades } \\
\text { recreativas } \\
\end{array}$ & \\
\hline & CSedu_04 & $\begin{array}{c}\text { Uso de aplicaciones de } \\
\text { videoconferencia (Zoom, } \\
\text { Google, Classroom, Microsoft } \\
\text { Teams) para actividades } \\
\text { educativas }\end{array}$ & \\
\hline \multirow[t]{3}{*}{$\begin{array}{c}\text { Salud física y } \\
\text { mental }\end{array}$} & CMcor_01 & $\begin{array}{l}\text { Realiza ejercicios de equilibrio } \\
\text { cuerpo y mente en la actividad } \\
\text { cotidiana. }\end{array}$ & \multirow[t]{3}{*}{3} \\
\hline & CMani_02 & Humor para mantener el ánimo & \\
\hline & CMopt_04 & $\begin{array}{l}\text { Optimismo sobre el desarrollo } \\
\text { y la evolución de la pandemia }\end{array}$ & \\
\hline \multirow[t]{3}{*}{$\begin{array}{c}\text { Responsabilidad } \\
\text { social }\end{array}$} & RScaus_01 & $\begin{array}{c}\text { Apoyo causas solidarias locales } \\
\text { y/o nacionales }\end{array}$ & \\
\hline & RSmarc_03 & $\begin{array}{c}\text { Elijo marcas de empresas } \\
\text { socialmente responsables con el } \\
\text { medio ambiente }\end{array}$ & 3 \\
\hline & RSprom_04 & $\begin{array}{l}\text { Promuevo e incentivo el } \\
\text { cumplimiento de la } \\
\text { responsabilidad social }\end{array}$ & \\
\hline
\end{tabular}

\section{Resultados}

Los resultados del análisis realizado sobre la medición y el modelo estructural, respaldado por el software Smart PLS es analizado a continuación.

Entre las características demográficas de los encuestados, es importante resaltar que la población está conformada por el $62 \%$ de mujeres; las edades de mayor representación en el estudio son universitarios entre 21 a 24 años, constituyendo el $46 \%$ de la muestra. El $75 \%$ son solteros y en su mayoría estudian en universidades públicas (55\%), mientras el $51 \%$ lo hacen a través de la modalidad a distancia (ver Table 2). 
Tabla 2. Variables demográficas

\begin{tabular}{|c|c|c|}
\hline Variables demográficas & Frecuencia & Porcentaje \\
\hline \multicolumn{3}{|c|}{ Género } \\
\hline Masculino & 152 & 38 \\
\hline Femenino & 253 & 62 \\
\hline \multicolumn{3}{|c|}{ Edad } \\
\hline 17 a 20 años & 58 & 14 \\
\hline 21 a 24 años & 187 & 46 \\
\hline 25 a 28 años & 69 & 17 \\
\hline 29 y más & 91 & 23 \\
\hline \multicolumn{3}{|c|}{ Estado civil } \\
\hline Soltero & 302 & 75 \\
\hline Casado & 69 & 17 \\
\hline Divorciado & 13 & 3 \\
\hline Unión libre & 20 & 5 \\
\hline Viudo & 1 & 0 \\
\hline \multicolumn{3}{|c|}{ Universidad } \\
\hline Pública & 223 & 55 \\
\hline Privada & 182 & 45 \\
\hline \multicolumn{3}{|c|}{ Modalidad de estudios } \\
\hline A Distancia & 207 & 51 \\
\hline Presencial & 198 & 49 \\
\hline
\end{tabular}

Algo que resaltar en la caracterización de la muestra, es la participación de estudiantes de universidades públicas y privadas, tanto de las modalidades presencial, abierta y a distancia; y on-line del país.

\section{Medición del modelo}

Luego de realizar las pruebas al modelo, se determinó que dos indicadores de la variable comportamiento de compra, cinco indicadores de la variable entretenimiento -factores personales-, un indicador del factor social, cuatro indicadores del factor de responsabilidad social; y, un indicador del factor psicológico -salud física y mental- no cumplieron con los test para este tipo de modelos; obteniendo, así un modelo con cuatro de las cinco variables planificadas y catorce de los veintiséis indicadores observados inicialmente.

Por su parte, los puntajes adecuados para las pruebas de confiabilidad compuesta y Cronbach's Alpha son >0.7, asegurando de esta manera una buena confiabilidad de los constructos. 
Tabla 3. Prueba Crombach's alpha

\begin{tabular}{|c|c|c|c|}
\hline Constructos- variables & $\begin{array}{c}\text { Dijkstra- } \\
\text { Henseler's rho } \\
\left(\mathbf{\rho}_{\mathbf{A}}\right)\end{array}$ & $\begin{array}{c}\text { Jöreskog's } \\
\text { rho (pc) }\end{array}$ & $\begin{array}{c}\text { Cronbach's } \\
\mathbf{a l p h a ( \alpha )}\end{array}$ \\
\hline Comp. Consum. Univ. & 0.7602 & 0.8461 & 0.7577 \\
\hline Relaciones Sociales & 0.7831 & 0.8367 & 0.7453 \\
\hline Responsabilidad social & 0.7074 & 0.8355 & 0.7042 \\
\hline Salud física y mental & 0.5996 & 0.7724 & 0.5685 \\
\hline
\end{tabular}

De acuerdo con lo establecido por Fornell y Larcker (1981) y Henseler et al. (2009), la validez convergente o Average Varieance Extracted (AVE) se encuentran sobre el valor 0.5 en todas las variables, lo que proporciona validez al modelo tal como se observa en la Table 4.

Tabla 4. Mediciones AVE

\begin{tabular}{|c|c|}
\hline Constructos & $\begin{array}{c}\text { Average variance } \\
\text { extracted (AVE) }\end{array}$ \\
\hline Comp. Consum. Univ. & 0.5792 \\
\hline Relaciones sociales & 0.5646 \\
\hline Responsabilidad social & 0.6291 \\
\hline Salud física y mental & 0.5345 \\
\hline
\end{tabular}

En todos los casos la validez convergente o Average Variance Extracted (AVE) es más alta que los valores de correlación presentados entre los constructos, lo que proporciona evidencia sobre una validez discriminante adecuada. Las mediciones del modelo proporcionan una buena evidencia sobre la confiabilidad y validez para representar los temas discutidos en este estudio (Tabla 5).

Tabla 5. Correlaciones entre variables (constructos)

\begin{tabular}{|c|c|c|c|c|}
\hline Constructos & $\begin{array}{c}\text { Comp. } \\
\text { Consum. Univ. }\end{array}$ & $\begin{array}{c}\text { Relaciones } \\
\text { Sociales }\end{array}$ & $\begin{array}{c}\text { Responsabilidad } \\
\text { social }\end{array}$ & $\begin{array}{c}\text { Salud } \\
\text { física y } \\
\text { mental }\end{array}$ \\
\hline Comp. Consum. Univ. & 0.5792 & & & \\
\hline Relaciones sociales & 0.2034 & 0.5646 & & \\
\hline Responsabilidad social & 0.2313 & 0.0610 & 0.6291 & \\
\hline Salud física y mental & 0.2688 & 0.2059 & 0.1430 & 0.5345 \\
\hline
\end{tabular}

Squared correlations; AVE in the diagonal.

\section{Modelo estructural}

El modelo desarrollado en este estudio se muestra en la Figura 1. Los resultados evidencian un buen índice SRMR (0.082), por debajo de los umbrales máximos de 0.10 ( 0.08 en el enfoque más conservador). 


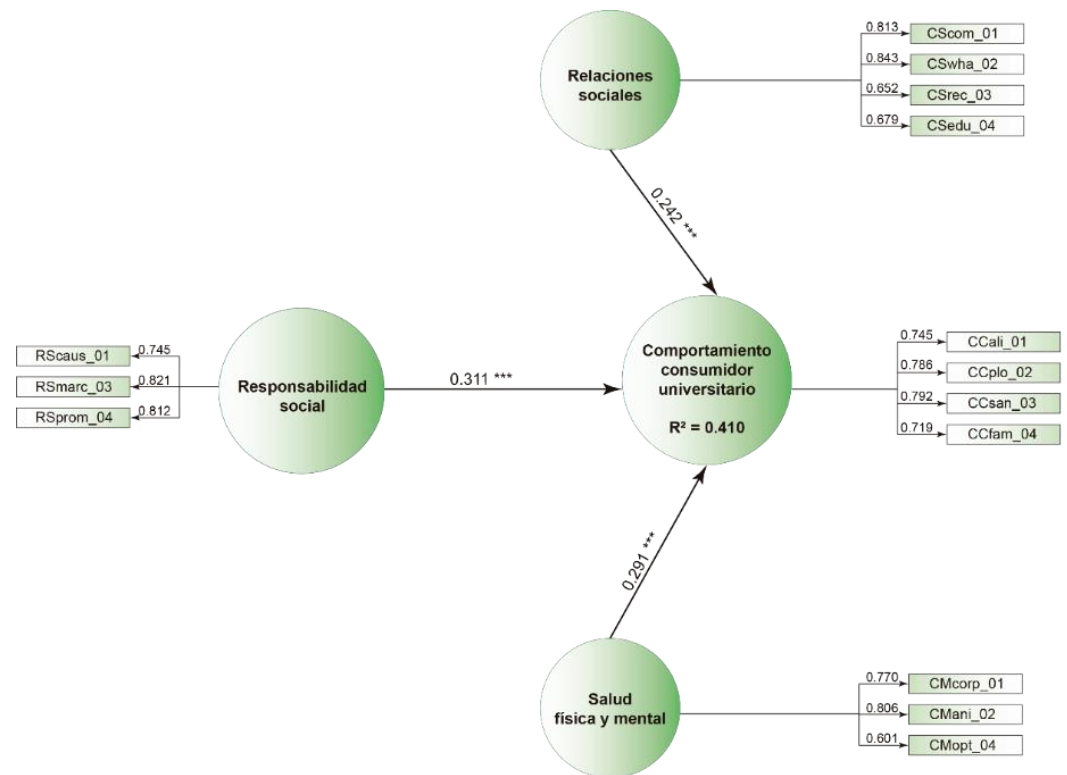

Figura 1. Modelo estructural del comportamiento del consumidor y Responsabilidad Social

La verificación de la consistencia del modelo medido a través del Standardized Root Mean Square Residual (SRMR) raíz cuadrada de la suma de diferencias al cuadrado entre las matrices de correlación -distancia euclidiana- (Bentler \& Yuan, 1999), muestran que el modelo de comportamiento del consumidor explica el $40 \%$ de las variables analizadas: factores sociales, responsabilidad social y psicológicos. Esta conclusión se basa en los valores $\mathrm{R}^{2}$ resultantes de 0.409 , que indican el porcentaje de variabilidad explicado por las construcciones predictivas en el modelo. Los valores $\mathrm{R}^{2}$ ajustados 0.405 "tienen en cuenta la complejidad del modelo y el tamaño de la muestra; $y$, por lo tanto, son para comparar diferentes modelos o el poder explicativo de un modelo en diferentes conjuntos de datos" (Henseler et al., 2016).

Por otro lado, en los estudios realizados por Churchill (1979) se establece que la confiabilidad del indicador debe encontrarse por arriba de 0.7. En la investigación los 14 indicadores presentan loading superiores al 0.7; así: CCcom_01 $>0.81$, CCwhat_02>0.84, RScaus_01>0.74, RSmarc_03>0.82, RSprom_04>0.81, CMcorp_01 $>0.76$, CMani_02 $>0.80$, CCali_01_02 $>0.74$, CCplo_02>0.78, CCsan_03 02>0.79 y CCfam_04>0.71; a excepción de CCrec_03<0.65, CSedu_04< 0.67; y, CMopt_04<0.60, que muestran valores cercanos a 0.7 lo cual no justifica su eliminación. Esta información confirma la bondad de los indicadores y la confiabilidad del instrumento.

A partir del $\mathrm{R}^{2}$ ajustado, el análisis de la evidencia empírica para las tres hipótesis planteadas $(\mathrm{H} 1),(\mathrm{H} 2)$ y $(\mathrm{H} 3)$, permiten señalar que la $\mathrm{H} 1$ : la relación entre el comportamiento del consumidor y factores sociales $(0.242, \mathrm{p}$ 
$<0: 01)$; H2: comportamiento del consumidor y la responsabilidad social (0.311, p <0:01); y; la H3: comportamiento de compra y factores psicológicos (salud) (0.291, p <0:01) son estadísticamente significativos, como se muestra en la Tabla 5.

Además, según la clasificación del tamaño del efecto $\left(\mathrm{f}^{2}\right)$ propuesta por Cohen, (2013), el modelo presenta una media $\mathrm{f}^{2}$ en las relaciones probadas de comportamiento del consumidor con las variables: relaciones sociales, responsabilidad social y factores psicológicos (salud), lo que proporciona buena evidencia sobre la previsibilidad del modelo (ver Tabla 6).

Tabla 6. Estimaciones, $p$ value y $f^{2}$ de Cohen's

\begin{tabular}{|c|c|c|c|c|c|c|}
\hline Hypothesis & Beta & $\begin{array}{c}\text { Standard } \\
\text { error }\end{array}$ & t-value & $\begin{array}{c}\text { p- } \\
\text { value }\end{array}$ & Cromba & $\begin{array}{c}\text { Cohen's } \\
\mathbf{f}^{2}\end{array}$ \\
\hline $\begin{array}{c}\text { H1: Relaciones Sociales-> } \\
\text { Comp. consumidor } \\
\text { universitario. }\end{array}$ & 0.2422 & 0.0517 & 4.6843 & 0.0000 & 0.7453 & 0.0782 \\
\hline $\begin{array}{c}\text { H2: Responsabilidad Social-> } \\
\text { Comp consumidor } \\
\text { universit }\end{array}$ & 0.3110 & 0.0414 & 7.5202 & 0.0000 & 0.7042 & 0.1393 \\
\hline $\begin{array}{c}\text { H3: Salud física y mental-> } \\
\text { Comp. consumidor } \\
\text { universitario. }\end{array}$ & 0.2910 & 0.0501 & 5.8099 & 0.0000 & 0.5685 & 0.1031 \\
\hline
\end{tabular}

\section{Discusión y conclusiones \\ Discusión}

La investigación analiza desde el Modelo de Ecuaciones Estructurales (SEM) el comportamiento del consumidor universitario a través de relaciones sociales, responsabilidad social y salud mental y física en tiempos de la crisis sanitaria producida por la COVID-19.

Estos resultados llevan a aceptar las hipótesis planteadas $(\mathrm{H} 1, \mathrm{H} 2$ y H3), de esta manera se determina que el comportamiento del consumidor de la población de estudio, está relacionado con los factores sociales, la responsabilidad social y los factores psicológicos.

A partir de ello, se puede reflexionar sobre cuatro hallazgos importantes:

i) Los estudiantes universitarios, pertenecientes al grupo de millennials y pos millennials, durante el periodo de aislamiento social han experimentado el apego familiar y sobre todo, el distanciamiento social con sus grupos de interés, estas barreras han sido superadas por el uso intensivo y variado de las redes sociales que han permitido de cierta manera mantenerse en contacto permanente, de manera virtual, con sus familiares y amistades.

ii) Si bien es cierto, el aislamiento durante la pandemia producida por la COVID-19 generó en un primer momento incertidumbre y sobre todo cambiar a otro tipo de contacto, como es el contacto virtual, esta 
propició también el consumo en línea. En este sentido, el grupo de estudio, con el uso de tecnologías y a través de las redes sociales y plataformas de la información, totalmente abiertas durante la pandemia, pudo acceder a la información, lo que hizo posible que su estilo de vida no se afecte y sobre todo facilito el camino al boom del consumo en línea, puesto que un gran número de oferentes de bienes y servicios llevó a cabo innovaciones para acercar los productos hacia el consumidor.

iii) Sin lugar a duda, durante la emergencia sanitaria, las personas en general han tomado más conciencia ecológica y esto se ha profundizado en el grupo de estudio, cuyas decisiones han sido más responsables con el daño ambiental. Cabe mencionar que los millennials y pos millennials son una generación involucrada con los problemas sociales y ambientales y por ello, al momento de consumir, revisan marcas, buscan tecnologías y enfocan su atención en la respuesta que las grandes empresas y corporaciones están llevando a cabo para apalear dichos problemas, en otras palabras, se preocupan por la responsabilidad social que existe detrás de cada empresa que ofrece un bien o servicio.

iv) Frente a estos cambios generados en tiempo de crisis, las empresas desean satisfacer las necesidades del grupo de millennials y pos millennials, con responsabilidad social. Ello implica, enfocarse a producir para satisfacer las necesidades de una población preocupada no solo de estar al día con las innovaciones; sino también, enfocada en el marco del respeto a los derechos humanos, cuidado y protección ambiental, y eliminación de la discriminación en las actividades sociales, económicas, culturales y ambientales.

\section{Conclusiones}

Esta investigación permitió diseñar un Modelo de Ecuaciones Estructurales (SEM) conformado por cuatro variables y catorce indicadores, validado estadísticamente a través de tres dimensiones: cargas de los indicadores, confiabilidad y validez convergente.

A través de esta metodología se pudo comprobar las hipótesis planteadas, concluyendo así, que existe una relación entre el comportamiento del consumidor universitario con las relaciones sociales, la responsabilidad social con las empresas y la salud mental y física.

De estas tres variables, los millennians y pos millennials, al momento de consumir, no solamente que revisa la satisfacción de sus necesidades, sino que analizan si la empresa que ofrece sus productos lleva a cabo actividades relacionadas con la responsabilidad social. Dentro de este marco, lo de mayor interés y valor para dicha generación es la responsabilidad social y 
medioambiental. En el primer caso relacionado con los temas de derechos del trabajador y cero discriminaciones laborales, en el segundo caso, se direcciona a producción que aplique procesos de reducción de degradación y/o contaminación al medio ambiente.

En este marco, la responsabilidad social se convierte en el elemento diferenciador para que una empresa perdure en el mercado, más aún en momentos como los actuales, en los que los efectos generados por la pandemia exigen que se analicen y revisen los procesos de producción y políticas empresariales. Pues para garantizar su rentabilidad, se deberá personalizar al consumidor, considerando sus derechos asociados a los del medio ambiente.

A partir de los resultados de la investigación, se abren algunas interrogantes para futuras investigaciones en el campo del marketing digital y responsabilidad social; temas cruciales en tiempos de crisis, considerando que el rol fundamental será el empoderamiento de los ciudadanos que habitan en espacios geográficos comunes, y que con un mayor ejercicio de conciencia individual y desde los entornos más cercanos, construyan una conciencia colectiva más ampliada, que permita mudar hacia verdaderas prácticas de consumo que garanticen crecimiento y desarrollo sostenible.

La investigación presentó algunas limitaciones en la recolección de información vía online, lo que disminuyó el número de observaciones y el alcance a un mayor número de universidades. Por lo que recomendamos ampliar esta investigación a otros contextos; y en el futuro realizar investigaciones que analicen el comportamiento de los consumidores de diferentes generaciones en tiempos de crisis y post crisis.

\section{References:}

1. Afifi, A. \& Clark, V. (1990). Computer-aided multivariate analysis. Nueva York: Chapman \& Hall.

2. Bentler, P. M. \& Yuan, K.H. (1999). Structural Equation Modeling with Small Samples: Test Statistics. Multivariate Behavioral Research, 34(2), 181-197. https://doi.org/10.1207/S15327906Mb340203

3. BID, Novelle, R., Repeto, A., \& Otros (2018). Millennials in Latin America and the Caribbean: to work or study? http://dx.doi.org/10.18235/0001411

4. Blackwell, R., Miniard, P., \& Engel, J. (2002). Comportamiento del Consumidor. México: Cengage Learning.

5. Cao, F., Wang, X., \& Wang, Z. (2020). Effects of awe on consumer preferences for healthy versus unhealthy food products. Journal of Consumer Behaviour, 19(3), 264-276. https://doi.org/10.1002/cb.1815

6. Caprariello, P. \& Reis, H. (2013). To do, to have, or to share? Valuing experiences over material possessions depends on the involvement of 
others. Journal of Personality and Social Psychology, 104(2), 199215. https://doi.org/10.1037/a0030953

7. Castelló, I., Castilla, M., \& Ibáñez, M. (2005). La actitud del consumidor hacia la Responsabilidad Social Corporativa (RSC). PriceWaterHouseCoopers.

8. https://jussemper.org/Newsletters/Resources/Actitud_Consum_Esp_ RSC.pdf

9. Churchill, G. (1979). A paradigm for Devoloping Better Measures of Marketing Construcs. Journal of Marketing Research, Vol16, 64-73. https://doi.org/10.1177/002224377901600110

10. Haenlein, M. \& Kaplan, A. (2004). A begginner's guide to partial least squares analysis. Understanding statistics. 3(4), 283-297.

11. Fornell, C. \& Larcker, D.F. (1981). Evaluating Structural Equation Models with Unobservable Variables and Measurement Error. Journal of Marketing Research, 18(1), 39-50. https://doi.org/10.1177/002224378101800104

12. Forte, M. \& Lamont, B.T. (1998). The Bottom Line Effects of Greening: Implications of Environmental Awarenes. Academy of Management, 12, 89-90.

13. Hair, Black, Babin \& Anderson (2020). Multivariate Data Analysis, 7th Edition | Pearson (s. f.). Recuperado 28 de mayo de, de https://www.pearson.com/us/higher-education/program/HairMultivariate-Data-Analysis-7th-Edition/PGM263675.html

14. Harrison, R. (2003). Corporate social responsibility and the consumer movement. Consumer Policy Review, 13(4), 127-131.

15. Henao, O. \& Córdova, J. (2007). Comportamiento del consumidor, una mirada sociológica. Red de Revistas Científicas de América Latina y el Caribe, España y Portugal, Colombia, 3(2), 17- 29. http://www.redalyc.org/pdf/2654/265420387003.pdf

16. Henseler, J., Hubona, G., \& Ray, P. A. (2016). Using PLS path modeling in new technology research: Updated guidelines. Industrial Management \& Data Systems, 116(1), 2-20. https://doi.org/10.1108/IMDS-09-2015-0382

17. Kreng B. \& Huang, M. (2011). Corporate Social Responsibility: Consumer behavior, corporate strategy and public policy. Social Behavior and Personality, 39, 529-542

18. Li, D. \& Atkinson, L. (2020). The role of psychological ownership in consumer happiness. Journal of Consumer Marketing, ahead-ofprint(ahead-of-print). https://doi.org/10.1108/JCM-09-2019-3420

19. Matas, A. \& Matas, A. (2018). Diseño del formato de escalas tipo Likert: Un estado de la cuestión. Revista electrónica de investigación educativa, 20(1), 38-47. 
20. Naciones Unidas (2020). Objetivos de desarrollo sostenible. https://www.un.org/sustainabledevelopment/es/sustainableconsumption-production/

21. Rajennd, M., Abdullah, Al-Mamun., P., Yukthamarani \& Noor, R. (2014). Factors Influencing Consumer Behavior: A Study among University Studentes in Malaysia. Asian Social Science, 10(9), 18-25.

22. Rodríguez, M. (2016). El consumo responsable y la responsabilidad social empresarial (RSE). Distribución y Consumo, 3, 56-163. https://www.nuevatribuna.es/media/nuevatribuna/files/2017/05/12/dy c_el_consumo_responsable_y_la_responsabilidad_social_empresarial -julio2016.pdf

23. Rodríguez, M. \& Bustamante, U. (2008). Desarrollo de competencias para el comportamiento ético-gerencial: un enfoque de responsabilidad. Cuadernos de Administración, 21(35), 205-208.

24. Sheth, J. (2020). Impact of COVID-19 on consumer behavior: Will the old habits return or die? Journal of Business Research, 117, 280-283. https://www.doi.org/10.1016/j.jbusres.2020.05.059

25. Schumacker, R.E. \& Lomax, R. G. (2010). A Beginner's Guide to Structural Equation Modeling (Third Edit). New York: Taylor \& Francis Group.

26. Solé, M. (2001). Los Consumidores del Siglo XXI. Madrid: Escuela Superior de Gestión Comercial y Marketing. ESIC, 2.

27. Su, C.-H. (Joan), Tsai, C.-H. (Ken), Chen, M.-H., \& Lv, W. Q. (2019). U.S. Sustainable Food Market Generation Z Consumer Segments. Sustainability, 11(13), 3607. https://doi.org/10.3390/su11133607

28. Xiao, G. \& Kim, J.O. (2009). The investigation of Chinese consumer values, consumption values, life satisfaction, and consumption behaviors. Psychology \& Marketing, 26(7), 610-624. https://doi.org/10.1002/mar.20291 